\title{
Jede CIRS-Meldung zählt!
}

\author{
Carmen Kerker-Speckera, Olga Frank ${ }^{\mathrm{b}}$ \\ ${ }^{a}$ MScN, Projektmanagerin, Patientensicherheit Schweiz; ${ }^{b}$ Dr., Projektleitung, Patientensicherheit Schweiz
}

Patientensicherheit Schweiz ist Trägerin des CIRRNET (Critical Incident Reporting \& Reacting NETwork), des überregionalen Netzwerks lokaler Fehlermeldesysteme Schweizer Gesundheitsinstitutionen, welches seit 2006 von Patientensicherheit Schweiz (= Stiftung für Patientensicherheit) betrieben wird. Im CIRRNET werden Meldungen aus lokalen Fehlermeldesystemen auf überregionalem Niveau vergemeinschaftet, den angeschlossenen Gesundheitsinstitutionen zugänglich gemacht und gemeinsam mit Experten bearbeitet. CIRRNET unterscheidet sich von den meisten CIRSNetzwerken dadurch, dass aus den lokalen Fehlermeldungen überregional relevante Problemfelder identifiziert und Verbesserungsempfehlungen von Fachleuten entwickelt und in Form der Quick-Alerts durch Patientensicherheit Schweiz verbreitet werden. Die lokalen Fehlermeldesysteme fördern die Sicherheitskultur und tragen zusammen mit dem verfügbaren Wissen der Quick-Alerts zur Verbesserung der Patientensicherheit bei. Sowohl die lokalen CIRS-Systeme als auch das CIRRNET leben vom Engagement aller Mitarbeitenden und bieten die Möglichkeit, aus Fehlern anderer zu lernen.

\section{CIRRNET-Meldemonat}

Patientensicherheit Schweiz setzt im Rahmen ihrer Ressourcen alles daran, den Nutzen für die am Netzwerk beteiligten Spitäler am CIRRNET zu erhöhen. Vom 1. bis 31. Oktober 2016 realisiert sie in diesem Zusammenhang erstmals in ihrem 10-jährigen Bestehen einen Meldemonat zu einem Hot-Spot in der Patientensicherheit, der «Fehletikettierung von Laborproben». In diesem Zeitraum sind alle Mitarbeitenden von CIRRNETSpitälern aufgerufen, alle kritischen Ereignisse, bei denen es zu einer Fehletikettierung von Laborproben gekommen ist, in ihrem lokalen CIRS zu erfassen. Gemeint sind sämtliche Laborproben, welche für Untersuchungszwecke bei Patienten abgenommen werden (z.B. Blut, Urin, Liquor, Abstriche, Biopsien etc.).

Von Interesse sind ganz konkret alle unerwünschten Ereignisse und die Umstände, wie bspw.

- falsche/fehlende Beschriftungen oder Etikettierungen von Entnahmeröhrchen, Laborzetteln, Laborresultaten,

- falsche/fehlende/verspätet eintreffende oder nicht auffindbare Laborresultate,
- Verwechslungen von Entnahmeröhrchen, Etiketten, Laborzetteln oder Laborresultaten und

- alles, was mit der Beschriftung und Etikettierung zu tun hat.

Beobachten Sie dazu einen unerwünschten Zwischenfall, dann berichten Sie Ihre Beobachtungen möglichst detailliert in Ihrem lokalen CIRS. Solche unerwünschten Zwischenfälle können beispielsweise im Rahmen einer Laborverordnung, der Entnahme oder dem Versand einer Laborprobe oder auch der Auswertung der Laborprobe bzw. dem Versand der Ergebnisse auftreten. Dementsprechend sind auch viele verschiedene Mitarbeitende einer Institution von dieser Thematik betroffen. Durch die Dokumentation eines unerwünschten Zwischenfalls im CIRS liefern Sie wertvolles Wissen, das sonst nirgends dokumentiert ist. Scheuen Sie sich daher nicht, auch längst bekannte oder "vermeintlich banale» Zwischenfälle zu melden.

Die CIRS-Verantwortlichen der Institutionen leiten diese lokalen CIRS-Meldungen während des Meldemonats regelmässig anonymisiert an CIRRNET weiter Alle in diesem Meldemonat eingehenden CIRS-Fälle werden von Patientensicherheit Schweiz zusammen mit diversen Fachexperten verschiedener Institutionen systematisch analysiert und bearbeitet. Die Ergebnisse der Analyse und die entwickelten Arbeitsresultate werden den teilnehmenden Institutionen zur Verfügung gestellt sowie an der CIRRNET-Tagung vom 23.3.2017 präsentiert.

Ziel dieses Meldemonats ist es, möglichst alle unerwünschten Ereignisse im Zusammenhang mit der Fehletikettierung von Laborproben als CIRS-Fälle zu erfassen. Diese Daten liefern Informationen über die Ausprägung und die Relevanz dieses Sicherheitsproblems und ermöglichen, das Spektrum der Ereignisse besser beurteilen zu können.

Helfen auch Sie mit, das Problemfeld für Ihre Institution vollumfänglich zu erfassen. Jede einzelne CIRSMeldung trägt etwas zur Verbesserung der Patientensicherheit bei. Daher gilt das Motto: Jede CIRS-Meldung zählt!

Ansprechpartner in den CIRRNET-Spitälern sind die lokalen CIRS-Verantwortlichen und Qualitäts- und Risikomanager.

Weitere Informationen finden Sie unter: www.cirrnet.ch 